\title{
Reliability Analysis of the Wet-end in Underwater Guard System
}

\author{
ZHANG Qianqian ${ }^{1,}$ a , HUANG Qingqing ${ }^{1, b}$ \\ ${ }^{1}$ No.5200 Jindu Road, Minhang District, Shanghai, China, 201108 \\ azhangqq_pretty@foxmail.com, 'bqq222300@163.com
}

Keywords: underwater, reliability, k/n Redundancy, hazard rate

\begin{abstract}
This paper presents the reliability analysis of the wet-end in underwater guard system. The difficulties in using active sonar in an underwater guard system include high reliability and the maintenance ability. The reliability block diagram of the system and main components was modeling, and the hazard rate of each PCB or Channel was predicted by part stress analysis. The reliability of different $\mathrm{k} / \mathrm{n}$ redundancy configurations was calculated and compared with, then optimized the configuration of the component.
\end{abstract}

\section{Introduction}

It was suggested that a significant terrorist threat might be posed to domestic harbors in the form of an explosive device delivered underwater by a scuba diver. The underwater targets can be detected by acoustic equipment in underwater guard system.[1] In order to keep high continuance and accuracy, the wet-end of the underwater guard system must have a very high reliability.

Reliability is defined as the probability that a system (component) will function over some time period $t$. To express this relationship mathematically we define the continuous random variable $T$ to be the time to failure of the system (component); $\mathrm{T} \geqslant 0$. Then the reliability can be expressed as

$$
R(t)=\mathrm{P}\{T \geq t\}
$$

Where $R(t) \geq 0, R(0)=1$, and $\lim _{t \rightarrow \infty} R(t)=0$. For a given value of $t, R(t)$ is the probability that the time to failure is greater than or equal to $t$. [2]

The wet-end reliability goal for an underwater guard system is 0.95 at 1 year, which means $R(8760 h)=0.95$.

\section{System Reliability Model}

\section{System Reliability block diagram}

The Wet-end in Underwater Guard System is composed of power module, energy-storage module, signal process module, power amplifier module, signal receive module, signal-conditioning module and signal transmit module. The reliability block diagram of the wet-end is as fig. 1.

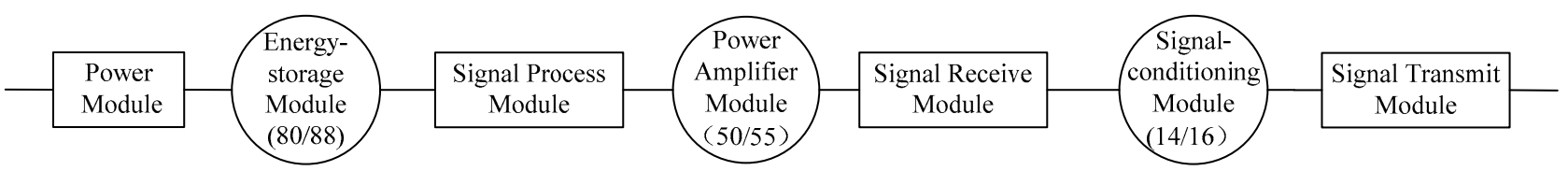

Fig. 1 reliability block diagram of the wet-end

The power module is composed of 3 PCBs (Printed Circuit Board) in parallel, which means that all PCBs must fail for the power module to fail. If one or more PCBs operate, the power module continues to operate.

All the redundancy modules in the system are designed to have $10 \%$ redundancy rate. The energy-storage module requires 80 out of its 88 energy-storage channels to operate in order to achieve orbit. The power amplifier module requires 50 out of its 55 channels function for the module to function. And the signal conditioning module requires 14 out of 16 channel to operate. 
The signal process module, signal receive model and signal transmit model are in series, which all components must function for the system to function.

Since wet-end reliability goal is 0.95 at 1 year, and there are 7 modules in the system, each module reliability equally to be $0.95^{1 / 7}=0.9927$.

\section{Reliability Prediction of Each Board}

The reliability of each component has been predicted by part stress analysis. And the results of the prediction are in table1.

Table 1 the reliability prediction results of each component

\begin{tabular}{|l|l|l|l|c|}
\hline Name & $\begin{array}{l}\text { Hazard rate } \\
\left(10^{-6} \mathrm{~h}^{-1}\right)\end{array}$ & Type & $\begin{array}{l}\text { Mission } \\
\text { Time(h) }\end{array}$ & $\begin{array}{c}\text { Mission } \\
\text { Reliability }\end{array}$ \\
\hline Power Board & 5.6878 & parallel (3) & 8760 & $\mathrm{R}_{1}$ \\
\hline Energy-storage Channel & 2.1565 & $80 / 88$, Redundancy & 8760 & $\mathrm{R}_{2}$ \\
\hline Signal Process Module & 0.7023 & serial, exponential & 8760 & $\mathrm{R}_{3}$ \\
\hline Power Amplifier Channel & 1.7789 & $50 / 55$ Redundancy & 8760 & $\mathrm{R}_{4}$ \\
\hline Signal Receive Module & 0.3495 & serial, exponential & 8760 & $\mathrm{R}_{5}$ \\
\hline Signal Conditioning Channel & 0.6610 & serial, exponential & 8760 & $\mathrm{R}_{6}$ \\
\hline Signal Transmit Module & 0.5641 & serial, exponential & 8760 & $\mathrm{R}_{7}$ \\
\hline
\end{tabular}

From the table 1, signal process module, signal receive module, and signal transmit module are in series. And the component failures are governed by the exponential failure law, then the reliability of the component can be calculated from Eq. 1. [3]

$$
R(t)=\exp \left(-\int_{0}^{t} \lambda d t^{\prime}\right)=e^{-\lambda t}
$$

$\lambda$, stand for the hazard rate. Obviously, the result of $\mathrm{R}_{3}, \mathrm{R}_{5}, \mathrm{R}_{6}$ and $\mathrm{R}_{7}$ can be calculated by Eq. $2-5$.

$$
\begin{aligned}
& R_{3}(8760)=e^{-\lambda_{3} t}=e^{-0.7023 \times 10^{-6} \times 8760}=0.9939 \\
& R_{5}(8760)=e^{-\lambda_{5} t}=e^{-0.3495 \times 10^{-6} \times 8760}=0.9969 \\
& R_{6}(8760)=e^{-\lambda_{6} t}=e^{-0.6610 \times 10^{-6} \times 8760}=0.9942 \\
& R_{7}(8760)=e^{-\lambda_{7} t}=e^{-0.5641 \times 10^{-6} \times 8760}=0.9951
\end{aligned}
$$

The power module has 3 power boards in parallel, and each board is the same. The reliability of the power module can be calculated by Eq. 6 .

$$
R_{1}(8760)=1-\left(1-e^{-\lambda_{1} t}\right)^{3}=1-\left(1-e^{-5.6878 \times 10^{-6} \times 8760}\right)^{3}=0.9999
$$

The energy-storage module and power amplifier module are in $\mathrm{k} / \mathrm{n}$ redundancy, the reliability analysis of $R_{2}$, and $R_{4}$ will be analysis particularly in chapter 3 of this paper.

\section{k/n Redundancy Reliability Analysis}

\section{k/n Redundancy Reliability Model}

A generalization of $n$ parallel components occurs when a requirement exists for $k$ out of $n$ identical and independent components to function for the system to function. The reliability block diagram of $\mathrm{k} / \mathrm{n}$ redundancy is as fig. 2 . 


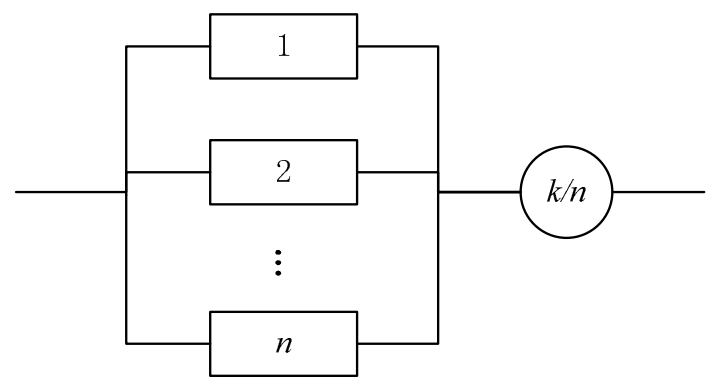

Fig.2 reliability block diagram of $\mathrm{k} / \mathrm{n}$ redundancy

Obviously $k \leqslant$ n. If $k=1$, complete redundancy occurs, and if $k=n$, the $n$ components are, in effect, in series. The reliability can be obtained from the binomial probability distribution.

If each component is viewed as an independent trial with $R(t)$ (its reliability) as a constant probability of success, then

$$
P(i)=C_{n}^{i} R^{i}(t)(1-R(t))^{n-i}
$$

is the probability of exactly $i$ components operating. $C_{n}^{i}$ is the number of ways (arrangements) in which $i$ successes (non-failures) can be obtained from $n$ components. $R^{i}(1-R)^{n-i}$ is the probability of $i$ success and $n-i$ failures for a single arrangement of successes and failures. Therefore

$$
R_{s}(t)=\sum_{i=k}^{n} P(i)=\sum_{i=k}^{n} C_{n}^{i} R^{i}(t)(1-R(t))^{n-i}
$$

In each module of the wet-end, the hazard rate of each channel is governed by exponential law, but hazard rate of the module would no longer be constant as it is a complex configuration, which is not in series. So the MTBF(Mean Time Between Failure) of the module isn't the multiplicative inverse of the hazard rate, it can be predicted by Eq. 9 .

$$
M T B F=\int_{0}^{\infty} R(t) d t=\int_{0}^{\infty} \sum_{i=k}^{n} C_{n}^{i} e^{-i \lambda t}\left(1-e^{-\lambda t}\right)^{n-i} d t
$$

Since

$$
\begin{aligned}
\left(1-e^{-\lambda t}\right)^{n-i} & =C_{n-i}^{0}(-1)^{n-i}\left(e^{-\lambda t}\right)^{n-i}+C_{n-i}^{1}(-1)^{n-i-1}\left(e^{-\lambda t}\right)^{n-i-1}+\cdots+C_{n-i}^{n-i}(-1)^{0}\left(e^{-\lambda t}\right)^{0}, \\
& =C_{n-i}^{0}(-1)^{n-i} e^{-n \lambda t+i \lambda t}+C_{n-i}^{1}(-1)^{n-i-1} e^{-n \lambda t+i \lambda+\lambda t}+\cdots+C_{n-i}^{n-i}
\end{aligned},
$$

The Eq. 9 can be infferred as Eq. 11 .

$$
\begin{aligned}
\text { MTBF } & =\int_{0}^{\infty} R(t) d t=\int_{0}^{\infty} \sum_{i=k}^{n} C_{n}^{i} e^{-i \lambda t}\left(1-e^{-\lambda t}\right)^{n-i} d t \\
& =\int_{0}^{\infty} \sum_{i=k}^{n} C_{n}^{i} e^{-i \lambda t}\left[C_{n-i}^{0}(-1)^{n-i} e^{-n \lambda t+i \lambda t}+C_{n-i}^{1}(-1)^{n-i-1} e^{-n \lambda t+i \lambda+\lambda t}+\cdots+C_{n-i}^{n-i}\right] d t \\
& =\sum_{i=k}^{n} C_{n}^{i}\left[C_{n-i}^{0}(-1)^{n-i} \int_{0}^{\infty} e^{-n \lambda t} d t+C_{n-i}^{1}(-1)^{n-i-1} \int_{0}^{\infty} e^{-n \lambda t+\lambda t} d t+\cdots+C_{n-i}^{n-i} \int_{0}^{\infty} e^{-i \lambda t} d t\right] \\
& =\sum_{i=k}^{n} C_{n}^{i}\left(C_{n-i}^{0}(-1)^{n-i} \frac{1}{-n \lambda}+C_{n-i}^{1}(-1)^{n-i-1} \frac{1}{(-n+1) \lambda} d t+\cdots+C_{n-i}^{n-i} \frac{1}{-i \lambda}\right)
\end{aligned}
$$

\section{Reliability Prediction of the Power Amplifier Module}

The power amplifier module requires 50 out of its 55 channels function for the module to function, and the reliability block diagram of the module is as fig.3. 


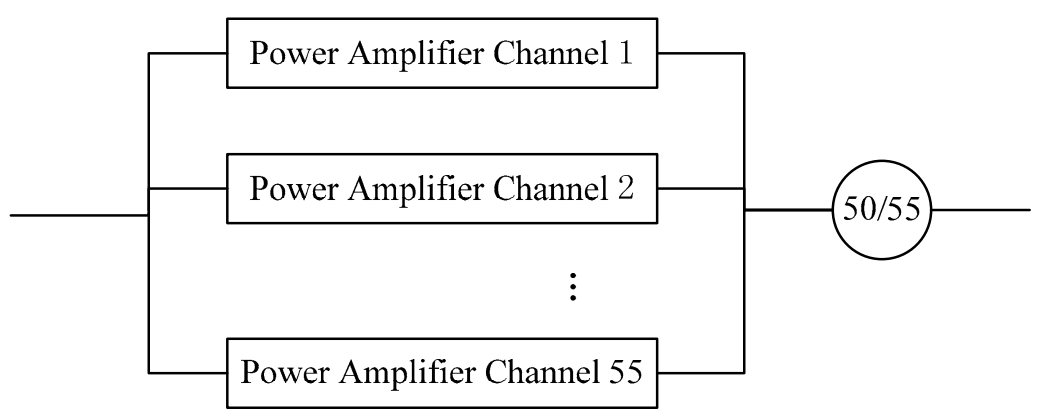

Fig.3 reliability block diagram of power amplifier module

As each channel is same and the failure distribution is exponential, the hazard rate of each channel is constant, the reliability of each channel can be calculate from Eq. 1. When $t=8760$, $\lambda_{4 \mathrm{c}}=1.7789 \times 10^{-6} h^{-1}$, the reliability of each power amplifier channel is $R_{4 c}(8760)=e^{-\lambda_{4 c} t}=0.9845$.

The reliability of $\mathrm{k} / \mathrm{n}$ redundancy can be calculate from Eq. 8 , and the reliability of the module is $\mathrm{R}_{4}$ as Eq. 12.

$$
R_{4}(t)=\sum_{i=k}^{n} C_{n}^{i} R^{i}(t)(1-R(t))^{n-i}=\sum_{i=50}^{55} C_{55}^{i} R_{4 c}^{i}(t)\left(1-R_{4 c}(t)\right)^{55-i}
$$

Using Matlab program can get the result of Eq. 12. is $R_{4}(8760)=0.9998$. Then the MTBF of the power amplifier module can be calculated by Eq. 13 .

$$
M T B F=\sum_{i=50}^{55} C_{55}^{i}\left(C_{55-i}^{0}(-1)^{55-i} \frac{1}{-55 \lambda_{4 c}}+C_{55-i}^{1}(-1)^{55-i-1} \frac{1}{(-55+1) \lambda_{4 c}} d t+\cdots+C_{55-i}^{55-i} \frac{1}{-i \lambda_{4 c}}\right)
$$

The result for the MTBF of the power amplifier module that can be generated through computer programs is $64313 h$.

\section{Optimization of the Configuration of the Energy-storage Module}

The energy-storage module requires 80 out of its 88 energy-storage channels function for the module to function. Since the hazard rate of each channel is $2.1565 \times 10^{-6} h^{-1}$, follow the Eq. 8 and Eq. 11, can get the reliability and MTBF for the $80 / 88$ redundancy configuration. Besides, as the module requires 80 channels to function, the results when total channels from 85 to 91 are compared in table 2 .

Table 2 the prediction for different kinds of redundancy

\begin{tabular}{|c|c|c|c|}
\hline No. & $\begin{array}{c}\mathrm{k} / \mathrm{n} \text { redundancy } \\
\text { Type }\end{array}$ & $\begin{array}{c}\text { Mission } \\
\text { Reliability }\end{array}$ & $\operatorname{MTBF}(\mathrm{h})$ \\
\hline 1 & $80 / 85$ & 0.9947 & 33739 \\
\hline 2 & $80 / 86$ & 0.9988 & 39131 \\
\hline 3 & $80 / 87$ & 0.9998 & 44461 \\
\hline 4 & $80 / 88$ & 0.9999 & 49726 \\
\hline 5 & $80 / 89$ & 0.9999 & 54900 \\
\hline 6 & $80 / 90$ & 0.9999 & 59030 \\
\hline 7 & $80 / 91$ & 0.9999 & 56211 \\
\hline
\end{tabular}

When energy-storage module is designed to have $10 \%$ redundancy- $80 / 88$ redundancy, the reliability of the module is 0.9999 . The reliability allocated equally to each module is 0.9927 , and the reliability of energy-storage module should be no less than the value. When the redundancy is $80 / 88$, the reliability is slightly higher, and that will be a waste for the system. From table 2, when choose $80 / 85$ redundancy, the reliability $\left(R_{2}(8760)=0.9947\right)$ is appropriate.

\section{System Reliability Prediction}

From the analysis presented above, we concluded the results of the reliability prediction in table 3. 
Table 3 the reliability prediction results of each module

\begin{tabular}{|l|l|l|l|}
\hline Name & $\begin{array}{l}\text { Hazard rate } \\
\left(10^{-6} \mathrm{~h}^{-1}\right)\end{array}$ & $\begin{array}{l}\text { Mission } \\
\text { Time(h) }\end{array}$ & $\begin{array}{l}\text { Mission } \\
\text { Reliability }\end{array}$ \\
\hline Power Board & 5.6878 & 8760 & 0.9999 \\
\hline Energy-storage Channel & 2.1565 & 8760 & 0.9947 \\
\hline Signal Process Module & 0.7023 & 8760 & 0.9939 \\
\hline Power Amplifier Channel & 1.7789 & 8760 & 0.9998 \\
\hline Signal Receive Module & 0.3495 & 8760 & 0.9969 \\
\hline Signal Conditioning Channel & 0.6610 & 8760 & 0.9942 \\
\hline Signal Transmit Module & 0.5641 & 8760 & 0.9951 \\
\hline
\end{tabular}

So we can predicts the reliability of the wet end

$$
R_{s}(8760)=\prod_{i=1}^{7} R_{i}=R_{1} R_{2} R_{3} R_{4} R_{5} R_{6} R_{7}=0.9748 .
$$

The result meets the requirement of the reliability goal, which is 0.95 at 1 year.

\section{Conclusions}

This paper discusses the mission reliability of the wet-end in underwater guard system, and the complexities associated with the $\mathrm{k} / \mathrm{n}$ redundancy. The presented method of the $\mathrm{k} / \mathrm{n}$ redundancy reliability prediction is based on the exponential failure law of each channel. Through the comparison of the different configuration of the $\mathrm{k} / \mathrm{n}$ redundancy, the energy-storage module and the power amplifier module are optimized. Then the reliability of the wet-end is predicted

\section{References}

[1] Brian Borowski, Alexander Sutin, Heui-Seol Roh, and Barry Bunin: Passive acoustic threat detection in estuarine environments, Optics and Photonics in Global Homeland Security IV, edited by Craig S. Halvorson, Daniel Lehrfeld, Theodore T. Saito Proc. of SPIE Vol. 6945, 694513, (2008) •

[2] Zhang Qianqian, Xu Feng, Huang Qingqing: Strength Reliability Analysis of a Lamella Structure based on Normal Distribution, Ship Electronic Engineering, No.12(2014)

[3] Charles E. Ebeling: An Introduction to Reliability and Maintainability Engineering (McGraw -Hill International Editions, 1997). 